\title{
NOTAS-PRÉVIAS
}

\section{NÍVEIS DE IRRIGAÇÃO NA GERMINAÇÃO DAS SEMENTES DE CENOURA ${ }^{1}$}

\author{
Francisco de Assis Lima Júnior ${ }^{2}$, Hugo Orlando Carvallo Guerra ${ }^{3}$ \& José Elias da Cunha Metri ${ }^{4}$
}

\begin{abstract}
RESUMO
Este trabalho foi desenvolvido na fazenda Cazuzinha, no Município de Sumé, Estado da Paraíba, com o propósito de se estudar o efeito de cinco níveis de água (irrigar diariamente com 20, 40, 60, 80 e $100 \%$ da evaporação medida no tanque "Classe A") durante o período de germinação das cultivares de cenoura Tropical e Brasília. As parcelas experimentais foram irrigadas com regadores comuns e o plantio realizado conforme as recomendações dos produtores locais. O delineamento estatístico foi o de blocos casualizados com arranjo fatorial $(5 \times 2)$ e três repetições. O efeito dos níveis de irrigação foi estatisticamente significativo a nível de $1 \%$ de probabilidade e a curva que melhor ajustou sua regressão foi: $y=-15,82+1,0542 x-0,00562 x^{2}$. Destacaram-se os tratamentos de 100 e $80 \%$ da evaporação no Tanque "Classe A" como os que proporcionaram as maiores germinações de semente, e o tratamento de $20 \%$ como o que produziu a menor germinação; nenhum efeito significativo foi observado para os tratamentos de cultivar, blocos e interação níveis versus cultivar. Para se obter boa germinação das cultivares Brasília e Tropical, quando cultivadas na microrregião homogênea do Cariri Ocidental paraibano, recomenda-se adotar níveis de irrigação com base em pelo menos $80 \%$ da evaporação medida no tanque "Classe A".
\end{abstract}

Palavras-chave: irrigação, evaporação, germinação, Daucus carota L.

\section{IRRIGATION LEVELS AND CARROT SEED GERMINATION}

\begin{abstract}
A study was conducted at Sumé County, State of Paraiba, Brazil, aiming to determine the effects of five water levels (daily irrigations with 20,40,60, 80 and $100 \%$ of the Class A Evaporation Pan) on the seed germination of two carrot cultivars (Tropical and Brasília). The experimental plots were planted according to local recommendations and irrigated with manual irrigators. The statistical layout was a factorial randomized block design $(5 \times 2)$ with three replicates. The effect of the water levels on the seed germination was statistically significant, at the $1 \%$ level of probability, and the regression curve obtained was $\mathrm{y}=-15.82+1.0542 \mathrm{x}-0.005621 \mathrm{x}^{2}$. The highest germination was obtained with the water management that replaced daily 80 and $100 \%$ of the water daily evaporated from Class A evaporation pan. The lowest germination was found when the irrigation replaced only $20 \%$ of the pan evaporation. No effect was observed for the cultivar treatment, blocks and for interaction. To obtain the best germination for both cultivars, when cropped in this region, it is recommended to irrigate daily with a water level equivalent to $80 \%$ of the Class A pan evaporation.
\end{abstract}

Key words: irrigation, evaporation, germination, Daucus carota L.

Recebido em 25/02/2000, Protocolo 021/00

${ }^{1}$ Parte da Dissertação de Mestrado do primeiro autor, apresentada ao Curso de Pós-Graduação em Engenharia Agrícola da Universidade Federal da Paraíba, Campus II, Campina Grande, PB

${ }^{2}$ Eng Agrônomo, M.Sc. em Engenharia Agrícola. Rua Joaquim da Silva Zeca 506, Santo Antônio, CEP 58100 - 360 , Campina Grande, PB. Fone: (0xx83) 321 2576.E-mail: falimajunior@zipmail.com.br

${ }^{3}$ Professor Ph.D. DEAg/CCT/UFPB. Rua Aprígio Veloso 882, Bodocongó, Campina Grande, PB. Fone: (0xx83) 310 1285, Fax: (0xx83) 310 1307.E-mail: hugo@deag.ufpb.br

${ }^{4}$ Professor Dr. DEAg/CCT/UFPB. Rua Aprígio Veloso, 882, Bodocongó, Campina Grande, PB. Fone: (0xx83) 310 1285, Fax:(0xx83) 310 1307.E-mail: metri@openline.com.br 


\section{INTRODUÇÃO}

A cenoura (Daucus carota L.), hortaliça da família umbelífera (Engler) ou apiácea (Cronquist) é cultivada há mais de dois mil anos, especialmente nas regiões de clima temperado, razão pela qual o é mais intensamente no Sudeste e no Sul do Brasil. A estimativa da área plantada no Brasil em 1996 foi da ordem de 16 mil hectares, com uma produção de 495 mil toneladas de raízes. Embora produza melhor em áreas de clima ameno, nos últimos anos, face ao desenvolvimento de cultivares tolerantes ao calor e com resistência às principais doenças de folhagem da cultura, o cultivo da cenoura tem-se expandindo também nos Estados da Bahia, Pernambuco e Distrito Federal (Makishima, 1997). No Nordeste brasileiro, as variedades mais recomendadas são as Tropical e Brasília, sendo que na Paraíba há maior aceitação pela variedade Tropical, bastante cultivada no sertão e nas áreas de temperaturas elevadas do Estado.

A cenoura é uma cultura cujas sementes germinam bem na faixa de temperatura entre 8 e $30^{\circ} \mathrm{C}$, sendo que a faixa ideal para obtenção de uma uniformidade maior do "stand" é em torno de 20 a $30{ }^{\circ} \mathrm{C}$, enquanto a temperatura de $35{ }^{\circ} \mathrm{C}$ a germinação é bastante reduzida e quase nula a $40{ }^{\circ} \mathrm{C}$. Nas condições de campo e na época mais quente do ano, a emergência ocorre entre cinco a sete dias após o semeio, enquanto no período mais fresco ocorre entre 10 a 12 dias (Pádua, et al. 1984).

Na maioria das hortaliças, na região de maior concentração de raízes, a umidade do solo deve ser mantida próximo à capacidade de campo, principalmente desde a fase de germinação até o fim do seu crescimento vegetativo; no caso da cenoura, recomenda-se uma umidade no solo entre 70 a $90 \%$ da disponível para as plantas, durante a fase de desenvolvimento vegetativo e formação das raízes mas, para a fase de maior carência, que ocorre durante e logo após a emergência das plântulas, tal teor deve ser mantido próximo aos 100\% desta água disponível (Filgueira, 1981). Segundo Bradley e Loudenslager (1971) a obtenção de estandes desejáveis de cenoura sempre tem sido um problema devido às diferenças em vigor e ao tamanho das sementes, encostramento do solo e alta susceptibilidade das sementes ao vento e altas temperaturas. Tentando pesquisar o efeito da irrigação sobre a diminuição da temperatura e conseqüentemente sobre a germinação das sementes, eles encontraram uma redução de 14 e 43\% na germinação, quando o solo foi irrigado semanalmente com 2,54 cm de água, e sem irrigação, respectivamente, quando comparado com o tratamento em que o solo era irrigado diariamente. Por outro lado, Hawthorn (1951) indica que a taxa de germinação é pouco influenciada pelo regime de umidade do solo. A fração de esgotamento limite ou limiar da água disponível para as plantas é um parâmetro extremamente importante para o manejo da irrigação de qualquer cultivo e, segundo Luján citado por Gomes (1994) para a cenoura este valor é de $40 \%$, ou seja, esta cultura tolera, no máximo, uma redução de $40 \%$ da sua água disponível total. Hargreaves (1975) recomenda que se aplique água ao solo quando já tiverem sido consumidos, pela planta, de 35 a 50\% da água disponível. Silva et al. (1982) indicam que, para se obter melhores produções de cenoura, deve-se manter uma alta freqüência de irrigação para que o potencial de água do solo se mantenha sempre elevado. Diversos métodos de irrigação podem ser utilizados na cultura da cenoura, sendo que a escolha do sistema deve ser baseada principalmente nos aspectos econômicos que nos propriamente técnicos. A irrigação por aspersão e por sulco é a mais utilizada, a subsuperficial e a por gotejamento são citadas por Marouelli \& Carrijo (1984) e o sistema de irrigação por mangueiras é citado por Soares \& Santos (1987). Este trabalho teve como objetivo identificar, no campo, o nível de irrigação com relação à evaporação observada no tanque "Classe A", que proporciona a melhor germinação das sementes das cultivares de cenoura, Tropical e Brasília.

\section{MATERIAL E MÉTODOS}

O experimento foi desenvolvido entre os dias 26 de setembro a 8 de outubro de 1998, na Fazenda "Cazuzinha", no Município de Sumé, Estado da Paraíba, Brasil. O delineamento estatístico adotado foi o de blocos casualizados, com arranjo fatorial $(5 \times 2)$ e 3 repetições, estudando-se o efeito de cinco níveis de irrigação (irrigar diariamente com 20, 40, 60, 80 e 100\% da evaporação observada no tanque "Classe A") na germinação das sementes de duas cultivares de cenoura. As características físicas do solo e a sua curva de retenção são apresentadas nas Tabelas 1 e 2, respectivamente. A água utilizada para a irrigação foi $\mathrm{C}_{2} \mathrm{~S}_{2}$, de acordo com a classificação proposta pelo US Salinity Laboratory Staff (United States, 1954). A unidade experimental constou de 30 parcelas, que mediam $2 \mathrm{~m}$ de comprimento e $1 \mathrm{~m}$ de largura, nas quais foram plantadas duas cultivares (Tropical e Brasília) de acordo com as recomendações dos irrigantes locais. Um pluviômetro e um tanque de evaporação "Classe A" foram instalados ao lado do experimento, de acordo com as normas das estações meteorológicas internacionais, segundo recomendações de Doorenbos e Pruitt (1976) e Doorenbos e Kassam (1979).

Tabela 1. Características físicas do solo

\begin{tabular}{|c|c|c|c|}
\hline \multirow{2}{*}{ Atributos } & \multicolumn{3}{|c|}{ Profundidade $(\mathrm{cm})$} \\
\hline & $0-15$ & $15-30$ & $30-45$ \\
\hline Areia $\left(\mathrm{g} \mathrm{kg}^{-1}\right)$ & 656,60 & 665,60 & 709,10 \\
\hline Silte $\left(\mathrm{g} \mathrm{kg}^{-1}\right)$ & 158,50 & 144,10 & 149,00 \\
\hline Argila $\left(\mathrm{g} \mathrm{kg}^{-1}\right)$ & 184,90 & 190,30 & 141,90 \\
\hline $\mathrm{Dg}^{*}\left(\mathrm{~g} \mathrm{~cm}^{-3}\right)$ & 1,41 & 1,41 & 1,42 \\
\hline $\mathrm{Dp}^{* *}\left(\mathrm{~g} \mathrm{~cm}^{-3}\right)$ & 2,64 & 2,67 & 2,50 \\
\hline Porosidade (\%) & 46,6 & 47,2 & 43,2 \\
\hline Classe Textural & F. Arenoso & F. Arenoso & F. Arenoso \\
\hline
\end{tabular}

Tabela 2. Retenção da água na camada de 0-15, 15-30 e 30-45 cm do solo

\begin{tabular}{cccc}
\hline \multirow{2}{*}{$\begin{array}{c}\text { Tensão } \\
(\mathrm{kPa})\end{array}$} & \multicolumn{3}{c}{ Conteúdo de água do solo $\left(\mathrm{cm}^{3} \mathrm{~cm}^{-3}\right)$} \\
\cline { 2 - 4 } & $0-15$ & $15-30$ & $30-45$ \\
\hline 0 & 0,47 & 0,47 & 0,43 \\
10,13 & 0,22 & 0,23 & 0,20 \\
33,43 & 0,12 & 0,12 & 0,12 \\
101,30 & 0,08 & 0,08 & 0,08 \\
506,50 & 0,07 & 0,07 & 0,07 \\
1519,50 & 0,06 & 0,06 & 0,05 \\
\hline
\end{tabular}

O semeio foi realizado em covas de $2 \mathrm{~cm}$ de profundidade, com espaçamentos de $20 \mathrm{~cm}$ entre fileiras e $5 \mathrm{~cm}$ entre covas. 
Com o auxílio de uma grade de madeira composta de marcadores, obtiveram-se a profundidade e os espaçamentos desejados (Fig. 1); depois, semeou-se uma semente por cova, num total de 200 por parcela, e se analisou o percentual de germinação das duas cultivares em estudo. $\mathrm{O}$ semeio foi feito após o solo ter sido levado à capacidade de campo; após a semeadura, cobriram-se as parcelas com esterco caprino curtido, devidamente peneirado.

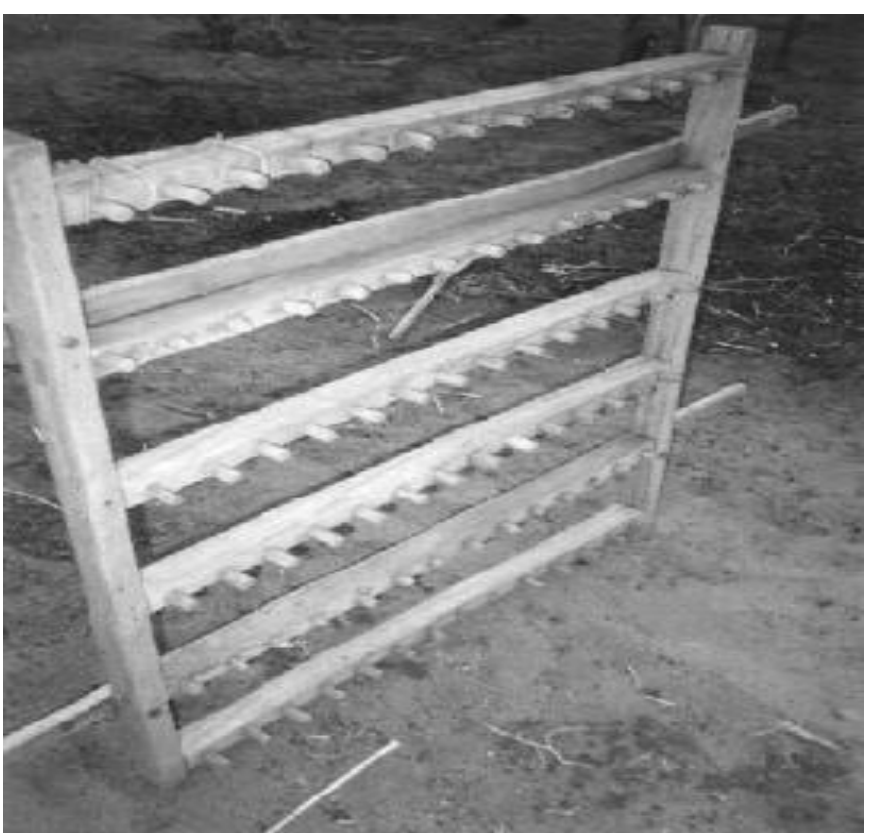

Figura 1. Grade de madeira para marcação das covas

Para obtenção do percentual de germinação fizeram-se contagens diárias das plântulas, sempre às $16: 30 \mathrm{~h}$, a partir do $7^{\circ}$ dia do semeio até o $12^{\circ}$, quando a germinação se estabilizou. As leituras diárias da evaporação no tanque "Classe A", obtidas em milímetros, foram realizadas às 7:00 $\mathrm{h}$, por meio de um micrômetro de gancho acoplado a um poço tranquilizador e serviam para o cálculo das lâminas de irrigação. A água foi aplicada diariamente através de um regador comum, manual e uniformemente no campo. Não houve chuvas durante o experimento de campo.

\section{RESULTADOS E DISCUSSÃO}

As lâminas de irrigação aplicadas em cada tratamento são apresentadas na Tabela 3.

Os resultados percentuais da germinação das cultivares de cenoura estudadas (Tropical e Brasília) obtidos em função dos níveis de irrigação adotados no trabalho, são apresentados na Tabela 4.

Observa-se, na Tabela 4, que as percentagens de germinação obtidas nessas cultivares, em todos os tratamentos, foram muito baixas quando comparadas com os índices de germinação indicados pelas empresas produtoras dessas sementes (as percentagens mínimas de germinação apontadas pelas empresas produtoras das sementes utilizadas neste trabalho, foram de 80 e 91\% para as cultivares Tropical ${ }^{1}$ e Brasília $^{2}$, respectivamente).

Isla Pak. Importadora de Sementes para Lavouras. Porto Alegre, RS

${ }^{2}$ Feltrin Importadora de Sementes Ltda. Farroupilha, RS
Tabela 3. Lâminas de irrigação utilizadas

\begin{tabular}{|c|c|c|c|c|c|c|}
\hline \multirow{2}{*}{\multicolumn{2}{|c|}{$\begin{array}{c}\text { Irrigação } \\
\text { e Data }\end{array}$}} & \multicolumn{5}{|c|}{ Lâmina Bruta Aplicada $^{*}(\mathrm{~mm})$} \\
\hline & & $\mathrm{T}_{100}$ & $\mathrm{~T}_{80}$ & $\mathrm{~T}_{60}$ & $\mathrm{~T}_{40}$ & $\mathrm{~T}_{20}$ \\
\hline 1 & $26 / 09 / 98$ & 10,98 & 8,78 & 6,59 & 4,39 & 2,20 \\
\hline 2 & $27 / 09 / 98$ & 10,19 & 8,15 & 6,11 & 4,08 & 2,04 \\
\hline 3 & $28 / 09 / 98$ & 11,50 & 9,20 & 6,90 & 4,60 & 2,30 \\
\hline 4 & 29/09/98 & 11,24 & 8,99 & 6,74 & 4,50 & 2,25 \\
\hline 5 & $30 / 09 / 98$ & 11,00 & 8,80 & 6,60 & 4,40 & 2,20 \\
\hline 6 & $01 / 10 / 98$ & 10,80 & 8,64 & 6,48 & 4,32 & 2,16 \\
\hline 7 & $02 / 10 / 98$ & 11,20 & 8,96 & 6,72 & 4,48 & 2,24 \\
\hline 8 & $03 / 10 / 98$ & 10,98 & 8,78 & 6,59 & 4,39 & 2,20 \\
\hline 9 & $04 / 10 / 98$ & 11,42 & 9,14 & 6,85 & 4,57 & 2,28 \\
\hline 10 & $05 / 10 / 98$ & 9,98 & 7,98 & 5,99 & 3,99 & 2,00 \\
\hline 11 & $06 / 10 / 98$ & 11,98 & 9,58 & 7,19 & 4,79 & 2,40 \\
\hline 12 & $07 / 10 / 98$ & 12,75 & 10,20 & 7,65 & 5,10 & 2,55 \\
\hline Total & & 134,02 & 107,22 & 80,41 & 53,61 & 26,80 \\
\hline
\end{tabular}

Tabela 4. Germinação da cenoura para os níveis de irrigação estudados

\begin{tabular}{|c|c|c|c|c|c|}
\hline \multirow{2}{*}{ Cultivar } & \multicolumn{5}{|c|}{ Germinação ${ }^{*}(\%)$} \\
\hline & $\mathrm{T}_{100}$ & $\mathrm{~T}_{80}$ & $\mathrm{~T}_{60}$ & $\mathrm{~T}_{40}$ & $\mathrm{~T}_{20}$ \\
\hline Tropical & 34 & 34 & 29 & 15 & 4 \\
\hline Brasília & 32 & 31 & 29 & 16 & 4 \\
\hline
\end{tabular}

A diferença encontrada pode ter acontecido devido ao fato de que os testes realizados pelas empresas foram feitos em laboratório, enquanto no presente estudo foram realizados diretamente no campo; assim, é possível que as baixas percentagens de germinação obtidas no presente trabalho estejam relacionadas a alguns fatores adversos, como a alta temperatura do local do trabalho (média $>30^{\circ} \mathrm{C}$ ) e/ou, ainda, pela formação de crostas sobre a superfície dos canteiros, ocasionada pelas altas temperaturas e pelos ventos fortes. Bradley e Loudenslager (1971), Murayama (1983) e Pádua et al. (1984) fazem referências sobre o efeito desses fatores na germinação das sementes de cenoura e justificam, de certa forma, a baixa germinação encontrada no presente estudo. Por outro lado, o tempo transcorrido entre os testes pode, também, ter contribuído para que os resultados não fossem reproduzidos. A temperatura, umidade relativa e a velocidade do vento da área do estudo, são apresentadas na Tabela 5.

Tabela 5. Temperatura, umidade relativa e velocidade do vento durante a germinação

\begin{tabular}{|c|c|c|c|c|}
\hline \multirow[t]{2}{*}{ Data } & \multirow{2}{*}{$\begin{array}{l}\text { Temperatura } \\
\text { Máxima } \\
\left({ }^{\circ} \mathrm{C}\right)\end{array}$} & \multicolumn{2}{|c|}{$\begin{array}{c}\text { Umidade } \\
\text { Relativa }(\%)\end{array}$} & \multirow{2}{*}{$\begin{array}{l}\text { Velocidade } \\
\text { do Vento } \\
\left(\mathrm{km} \mathrm{dia}^{-1}\right)\end{array}$} \\
\hline & & $9: 00 \mathrm{~h}$ & $18: 00 \mathrm{~h}$ & \\
\hline $26 / 09 / 98$ & 30,20 & 87,52 & 59,04 & 183,00 \\
\hline 27/09/98 & 30,60 & 96,62 & 69,47 & 327,60 \\
\hline $28 / 09 / 98$ & 31,60 & 91,92 & 59,66 & 292,20 \\
\hline 29/09/98 & 32,00 & 90,89 & 64,85 & 238,30 \\
\hline $30 / 09 / 98$ & 31,40 & 93,37 & 63,63 & 199,10 \\
\hline $01 / 10 / 98$ & 30,20 & 87,52 & 59,04 & 186,80 \\
\hline $02 / 10 / 98$ & 30,60 & 81,30 & 67,61 & 231,60 \\
\hline 03/10/98 & 31,20 & 80,53 & 55,57 & 232,00 \\
\hline 04/10/98 & 31,00 & 95,07 & 65,86 & 346,50 \\
\hline $05 / 10 / 98$ & 31,20 & 93,38 & 57,17 & 271,20 \\
\hline $06 / 10 / 98$ & 30,60 & 95,07 & 64,25 & 266,90 \\
\hline $07 / 10 / 98$ & 31,20 & 89,27 & 59,19 & 265,80 \\
\hline Média & 30,98 & 90,21 & 62,11 & 253,42 \\
\hline
\end{tabular}


A análise de variância identificou que não houve diferença significativa entre blocos, cultivares e interação cultivar $\mathrm{x}$ irrigação, mas ocorreu efeito significativo a nível de $1 \%$ de probabilidade, entre a germinação de ambas as cultivares, para os tratamentos de irrigação estudados. A não significância encontrada entre blocos é devida, provavelmente, a fatores relacionados com a homogeneidade do solo da área experimental, conforme a classificação textural apresentada na Tabela 1. Para as cultivares, a não significância apresentada nos resultados da análise pode ter acontecido devido a fatores relacionados à adaptação similar das cultivares, às condições climáticas e do solo da região estudada, conforme cita a Agroceres (1990) e/ ou, ainda, em razão da igualdade das práticas culturais a que ambas as cultivares foram submetidas.

A Tabela 6 apresenta a comparação entre as médias das percentagens de germinação das duas cultivares para os diferentes tratamentos de irrigação estudados, conforme o Teste de Tukey, a nível de $5 \%$ de probabilidade $(\mathrm{p} \leq 0,05)$.

Tabela 6. Percentagem de germinação média da cenoura em função de tratamentos de irrigação estudados

\begin{tabular}{cc}
\hline Tratamentos $^{*}$ & Germinação $^{* *}(\%)$ \\
\cline { 2 - 2 } & (Média das Cultivares de Cenoura) \\
\hline $\mathrm{T}_{100}$ & $33,07 \mathrm{a}$ \\
$\mathrm{T}_{80}$ & $32,67 \mathrm{a}$ \\
$\mathrm{T}_{60}$ & $28,71 \mathrm{~b}$ \\
$\mathrm{~T}_{40}$ & $15,20 \mathrm{c}$ \\
$\mathrm{T}_{20}$ & $3,84 \mathrm{~d}$ \\
\hline $\mathrm{T}_{100}, \mathrm{~T}_{80}, \mathrm{~T}_{60}, \mathrm{~T}_{40}$ e $\mathrm{T}_{20}-100,80,60,40$ e $20 \%$ da evaporação no tanque Classe A, respectivamente \\
${ }^{* *}$ Médias seguidas da mesma letra não diferem significativamente a nivel de $5 \%$ da probabilidade
\end{tabular}

De acordo com a Tabela 6, verifica-se que as médias das germinações obtidas com os tratamentos de 100 e $80 \%$ da evaporação no tanque Classe $\mathrm{A}$, não apresentaram diferenças significativas; no entanto, houve diferenças significativas entre as médias desses dois tratamentos, com relação as médias obtidas nos demais. Observam-se, ainda, diferenças significativas entre as médias dos outros três tratamentos (60, 40 e $20 \%$ da evaporação no tanque Classe A) e, desta forma, pode-se admitir que os tratamentos de 100 e $80 \%$ da evaporação no tanque classe $\mathrm{A}$, representam os melhores manejos de água durante a fase de germinação das cultivares estudadas, enquanto o tratamento de $20 \%$ da evaporação no tanque Classe A foi o que apresentou o mais baixo índice de germinação, e os demais apresentaram valores intermediários, decrescentes com relação aos primeiros.

A partir dos resultados da Tabela 6, elaborou-se a curva de regressão (Fig. 2) a qual é uma equação de segundo grau, significativa a nível de $1 \%$ de probabilidade, de acordo com a qual se observa que os tratamentos de 80 e $100 \%$ da evaporação no tanque Classe A apresentam valores de germinação muito próximos, com pontos localizados praticamente na mesma altura da curva. Tal fato reforça os resultados obtidos no teste de comparação das médias, visto anteriormente na Tabela 6 .

Levando-se em conta os resultados obtidos recomenda-se, para a região semi-árida do Cariri Ocidental paraibano, a utilização da cenoura cultivar Tropical, irrigada durante a germinação com lâminas equivalentes à reposição diária de $80 \%$ da evaporação no Tanque Classe A. Como na análise de variância

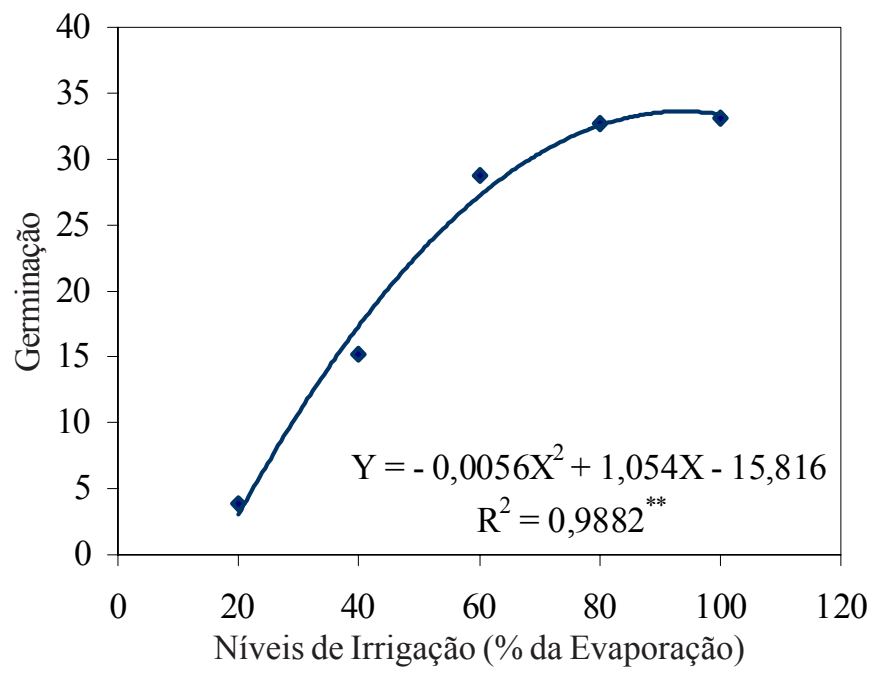

Figura 2. Germinação da cenoura para os cinco níveis de irrigação estudados

não houve efeito significativo entre as cultivares, a opção pela variedade Tropical se deu pelo fato da mesma ser a mais cultivada na região de estudo. Recomenda-se ademais, antes do estabelecimento da sementeira, tomar cuidados especiais com respeito a qualidade das sementes e características da sementeira para evitar baixa germinação.

\section{CONCLUSÃO}

Considerando-se o efeito significativo dos níveis de irrigação estudados sobre a germinação das sementes de cenoura, para se obter um bom stand de plântulas, em qualquer uma das duas cultivares estudadas (Tropical ou Brasília) nas condições climáticas da microrregião do Cariri Ocidental paraibano, recomenda-se irrigações diárias com lâminas de água equivalentes a $80 \%$ da evaporação observada no tanque "Classe A".

\section{REFERÊNCIAS BLIBIOGRÁFICAS}

AGROCERES, S. Paulo. Almanaque AGROCERES 1990. São Paulo, 1990. p.56-59.

BRADLEY, G.A.; LOUDENSLAGER, M.I. Stands, yields, and quality of irrigated carrots. Arkansas Farm Research, v.20, n.1, p.8, 1971.

DOORENBOS, J.; PRUITT, W.O. Crop and water requirements. Rome: FAO, 1976. Irrigation and Drainage Paper, 24

DOORENBOS, J.; KASSAM, A.H. Yield response to water. Rome: FAO, 1979. 193p. Irrigation and Drainage Paper, 33

FILGUEIRA, F.A.R. Manual de olericultura; cultura e comercialização de hortaliças. 2. ed. São Paulo: Agronômica Ceres, 1981.v. 1.1981.

GOMES, H.P. Engenharia de Irrigação; Hidráulica dos Sistemas Pressurizados, Aspersão e Gotejamento. João Pessoa, PB: Editora Universitária da UFPB. 1994. 344p.

HARGREAVES, G.H. Manual de requerimento de água para culturas irrigadas. Logan, Utah State University, 1975. 41p.

HAWTHORN, LR. Studies of soil moisture and spacing for seed production. Washington, D.C. Department of Agriculture, 1951.26p. 
MAKISHIMA, N. Cultivo da cenoura: colheita e comercialização. Instruções Técnicas da EMBRAPA № 13. Brasília. Dezembro 1997

MAROUELLI, W.A.;CARRIJO, O.A. Irrigação da cultura da cenoura e da mandioquinha-salsa. Informe Agropecuário, Belo Horizonte, MG. n.10, p.32-36, 1984.

MURAYAMA, S. Horticultura. Campinas: Instituto Campineiro de Ensino Agrícola, 1983. 321p. il.

PÁDUA, J.G. de; CASALI, V.W.D.; PINTO, C.M.F. Efeitos climáticos sobre a cenoura. Informe Agropecuário. Belo Horizonte, MG. n. 10, p. 11-13, 1984.
SILVA, W.L.C.; VIEIRA,J.V.; CARRIJO, O.A. Efeito de diferentes tensões de umidade do solo sobre a cultura da cenoura (Daucus carota L.) em fase de desenvolvimento do sistema radicular. Pesquisa Agropecuária Brasileira, Brasília, v.17, n.2, p.211-214, 1982.

SOARES, J.M.; SANTOS, E.D. Sistema de Irrigação por Mangueiras. ITEM. São Paulo, SP. n.28, p.21-24. 1987.

UNITED STATES. Salinity Laboratory Staff. Diagnosis and improvement of saline and alkaline soils. USDA. 1964. 160p. Agriculture Handbook $n^{\circ} 60$ 